\title{
1 Serum RNAs can predict lung cancer up to 10 years prior to diagnosis
}

2

3 Authors

4 Sinan Uğur Umu ${ }^{1,{ }^{*}}$, Hilde Langseth ${ }^{1,2}$,Verena Zuber $^{2}$, Åslaug Helland ${ }^{3,4,5}$, Robert Lyle ${ }^{6,7}$ and Trine B.

5 Rounge $\mathrm{e}^{1,8^{*}}$

6

7 Affiliations

$8{ }^{1}$ Department of Research, Cancer Registry of Norway, Oslo, Norway.

$9 \quad{ }^{2}$ Department of Epidemiology and Biostatistics, Imperial College London, London, UK.

$10{ }^{3}$ Department of Oncology, Oslo University Hospital, Oslo, Norway

$11{ }^{4}$ Institute for Cancer Research, Oslo University Hospital, Oslo, Norway

$12{ }^{5}$ Institute of Clinical Medicine, University of Oslo, Oslo, Norway

$13{ }^{6}$ Department of Medical Genetics, Oslo University Hospital and University of Oslo, Oslo, Norway

$14{ }^{7}$ Centre for Fertility and Health, Norwegian Institute of Public Health, Oslo, Norway

$15{ }^{8}$ Department of Informatics, University of Oslo, Oslo, Norway

17 Corresponding authors

18 Sinan U. Umu, sinan.ugur.umu@kreftregisteret.no, Ullernchausseen 64, 0379 Oslo, Norway

19 Telephone: +4722928906

20 Trine B. Rounge, trine.rounge@kreftregisteret.no, Ullernchausseen 64, 0379 Oslo, Norway

21 Telephone: +4722928734

22

23

24

25

26

27 


\section{Abstract}

Lung cancer (LC) prognosis is closely linked to the stage of disease when diagnosed. We investigated the biomarker potential of serum RNAs for the early detection of LC in smokers at different prediagnostic time intervals and histological subtypes. In total, 1061 samples from 925 individuals were analyzed. RNA sequencing with an average of 18 million reads per sample was performed. We generated machine learning models using normalized serum RNA levels and found that smokers later diagnosed with LC in 10 years can be robustly separated from healthy controls regardless of histology with an average area under the ROC curve (AUC) of $0.76(95 \% \mathrm{Cl}, 0.68-0.83)$. Furthermore, the strongest models that took both time to diagnosis and histology into account successfully predicted non-small cell LC (NSCLC) between 6 to 8 years, with an AUC of 0.82 (95\% Cl, 0.76-0.88), and SCLC between 2 to 5 years, with an AUC of $0.89(95 \% \mathrm{Cl}, 0.77-1.0)$, before diagnosis. The most important separators were microRNAs, miscellaneous RNAs, isomiRs and tRNA-derived fragments. We have shown that $L C$ can be detected years before diagnosis and manifestation of disease symptoms independently of histological subtype. However, the highest AUCs were achieved for specific subtypes and time intervals before diagnosis. The collection of models may therefore also predict the severity of cancer development and its histology. Our study demonstrates that serum RNAs can be promising prediagnostic biomarkers in a LC screening setting, from early detection to risk assessment.

(1)

48

9

5


64

65

66

67

68

69

70

71

72

73

74

75

76

77

78

79

80

81

82

83

84

85

86

87

88

89

90

91

92

93

94

95

96

97

98

\section{Introduction}

Lung cancer (LC) continues to be the leading cause of cancer-related deaths despite declining smoking prevalence (Bray et al., 2018; Wild et al., 2020). Non-small-cell (NSCLC) and small-cell (SCLC) are the two major subtypes of LC. The symptoms generally occur at a late stage and the prognosis is poor. Stage at diagnosis typically determines patient survival (Aberle et al., 2011; Bach et al., 2012; Brustugun et al., 2018). Screening with low-dose computed tomography (LDCT) can be effective for early detection (Bach et al., 2012; Peled and llouze, 2015) and reduce LC mortality up to $20 \%$ in high-risk groups (de Koning et al., 2020; Hanash et al., 2018; Seijo et al., 2019). However, LDCT has limitations such as high false-positive rates, risk of overdiagnosis and high costs (Gopal et al., 2010; Peled and llouze, 2015). Annual CT scans also cause harmful radiation exposure (Bach et al., 2012; Hanash et al., 2018). Robust biomarkers can help stratify high-risk groups and increase accuracy in patient inclusion criteria for LDCT-based screening programs (Hanash et al., 2018).

Liquid biopsies quantifying molecular biomarkers in circulation, such as tumor-derived DNAs, proteins and RNAs, and can be used to detect cancer (Hanash et al., 2018; Ko et al., 2018; Sandfeld-Paulsen et al., 2016). MicroRNAs (miRNA), a class of $\sim 21$ nucleotide long short RNAs, have been widely investigated for their biomarker potential (Fehlmann et al., 2020; Keller and Meese, 2016; Pichler and Calin, 2015; Tian et al., 2019). They can be found both in serum (Keller and Meese, 2016; Murillo et al., 2019; Umu et al., 2018) and plasma (Freedman et al., 2016; Keller and Meese, 2016; Murillo et al., 2019) as cell-free circulating RNAs, which may originate from dying cells or be actively secreted (Zaporozhchenko et al., 2018). Some of them are bounded by proteins or confined in layered exosomes which can protect them from degradation (Fritz et al., 2016). MiRNAs can function as tumor suppressors or oncomiRs and regulate tumor traits such as cell growth, angiogenesis, immune evasion and metastasis (Pichler and Calin, 2015; Svoronos et al., 2016). The search for RNA biomarkers is not limited to miRNAs. Aberrant expression of other RNA classes, such as protein coding mRNAs, tRNAs, piwi-interacting RNAs (piRNAs) and long-noncoding RNAs (IncRNAs), have been associated with cancer (Kim et al., 2017; Slack and Chinnaiyan, 2019). Despite the immense potential of cell-free RNAs, the promise of non-invasive RNA biomarkers of cancer has not yet been fulfilled.

One explanation of the lack of circulating RNAs used in clinical settings is our limited understanding of the prediagnostic dynamics of cell-free RNAs, since studies are usually based on samples at or after diagnosis. Carcinogenesis is a multistep process that turns cell functions from normal to malignant (Hanahan and Weinberg, 2000). It can cause temporal changes in RNA levels linked to cellular processes driven by the hallmarks of cancer (Gutschner and Diederichs, 2012; Hanahan and 
Weinberg, 2000). We have shown that prediagnostic RNA levels in serum are highly dynamic in lung cancer patients, which may signal early carcinogenesis (Umu et al., 2019). A similar result was observed in breast cancer (Lund et al., 2016) and testicular cancer patients (Burton et al., 2020). A lack of reproducibility among studies is also a problem, caused by technical and biological factors such as storage time, sampling procedure, age, sex, smoking history etc. (Rounge et al., 2018). It is therefore important to control for these factors.

In the present study, our objective was to identify serum RNA based biomarkers for early diagnosis of LC using prediagnostic samples. We identified the optimal machine learning (ML) algorithm for RNA biomarker modeling. Optimization of prediction models was done with an ML workflow, including cross-validation and testing, which was repeated five times to increase the generalizability of our results. We also investigated the biological relevance of the best RNA separators in the context of cancer biomarkers.

\section{Results}

\section{Patient characteristics and RNA-seq profiles}

In this study, we selected 400 patients with prediagnostic serum samples including multiple samples from the same patients. We also included 525 individuals as controls. After excluding failed or low input samples, we obtained RNA-seq data from 1061 serum samples. However, samples from individuals without any smoking history (i.e. never smokers) or missing information were excluded from initial analyses. This resulted in 535 cases and 263 control samples from 645 current or former smokers for modelling and testing (Table 1 and Figure 1A). Non-smokers consist of 7 cases and 256 control samples from 260 individuals (Supplementary File 1). We used non-smokers in a leave-out set only to test our final models and to calculate relative risk (RR).

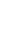


132 After filtering out low-count transcripts, 3306 RNAs were selected as candidate features and used in 133 the models: 202 miRNAs, 1137 isomiRs, 89 miscellaneous RNAs (miscRNAs), 380 piRNAs, 119 small 134 nucleolar RNAs (snoRNAs), 530 tRFs, 790 mRNAs and 59 IncRNAs.

135

136

137

138

139 Table 1. Clinical and histological characteristics of samples used in modelling.

\begin{tabular}{|c|c|c|c|c|c|}
\hline & \multicolumn{4}{|c|}{ Stage } & \multirow[b]{2}{*}{ Controls } \\
\hline & Early (Localized) & $\begin{array}{c}\text { Locally } \\
\text { Advanced } \\
\text { (Regional) }\end{array}$ & $\begin{array}{c}\text { Advanced } \\
\text { (Distant) }\end{array}$ & Unknown & \\
\hline \multicolumn{6}{|l|}{ Histology } \\
\hline NSCLC & 84 & 99 & 167 & 11 & - \\
\hline SCLC & 9 & 35 & 76 & 4 & - \\
\hline Others & 10 & 5 & 31 & 4 & - \\
\hline \multicolumn{6}{|l|}{ Sex } \\
\hline Male & 78 & 104 & 178 & 12 & 185 \\
\hline Female & 25 & 35 & 96 & 7 & 78 \\
\hline
\end{tabular}

\begin{tabular}{|c|c|c|c|c|c|}
\hline \multicolumn{6}{|l|}{$\begin{array}{l}\text { Age at donation, } \\
\text { yrs }\end{array}$} \\
\hline Mean (SD) & $54.3(7.33)$ & $54.9(9.08)$ & $53.5(8.25)$ & $51.8(6.53)$ & $49.9(10.9)$ \\
\hline \multicolumn{6}{|l|}{$\begin{array}{l}\text { Age at diagnosis, } \\
\text { yrs }\end{array}$} \\
\hline Mean (SD) & $59.8(7.67)$ & $60.6(8.89)$ & $59.4(8.31)$ & $58.6(6.05)$ & - \\
\hline \multicolumn{6}{|l|}{$\begin{array}{l}\text { Prediagnostic } \\
\text { sampling time, } \\
\text { yrs }\end{array}$} \\
\hline Mean (SD) & $5.52(2.81)$ & $5.63(2.79)$ & $5.91(2.66)$ & $6.75(2.18)$ & - \\
\hline Total Samples & 103 & 139 & 274 & 19 & 263 \\
\hline Individuals & 79 & 102 & 189 & 16 & 263 \\
\hline Total Individuals & & 645 & $\left.\mathrm{rs}^{*}\right)$ & & \\
\hline
\end{tabular}

$140 \quad{ }^{*}$ See supplementary document for non-smokers (Supplementary File 1). 


\section{ML algorithms can differentiate between prediagnostic cases and controls regardless of} prediagnostic time

145 We first evaluated the classification performance of the ML algorithms in terms of average AUCs on test datasets, created by five random repeats as explained in materials and methods.

147

148

149

150

151

152

153

154

155

156

157

158

159

160

161

162

163

164

165

166

167

168

169

170

171

172

173

174

175

176

All samples were included in algorithm evaluation regardless of their stage at diagnosis and prediagnostic time which were regarded as full-time standard models (Figure 2 - Figure supplement 1). The average AUC of all algorithms was $0.67(95 \% \mathrm{Cl}, 0.66-0.69)$ for all histologies, $0.67(95 \% \mathrm{Cl}$, 0.65-0.69) for NSCLC and $0.64(95 \% \mathrm{Cl}, 0.62-0.66)$ for SCLC on the test datasets. The XGBoost algorithm produced a higher AUC than the average, $0.71(95 \% \mathrm{Cl}, 0.68-0.73)$. The XGBoost models also performed better when the samples were stratified by histologies: NSCLC, $0.70(95 \% \mathrm{Cl}, 0.65$ 0.75), and SCLC, $0.71(95 \% \mathrm{Cl}, 0.68-0.74)$ (Figure 2).

Although the models of all algorithms had comparable performances in terms of average AUCs, they differ in terms of total number of non-zero features (i.e. different model complexity). For example, RF selected more than 3000 non-zero features while the lasso model selected fewer than 25 features. However, the profiles of the top features, ranked in terms of feature importance, usually consisted of similar RNAs (e.g. miRNAs or tRFs).

Since XGBoost produced the most predictive full-time models, we used it for the remaining analyses. We also investigated the best predictors of the XGBoost models and ranked them based on their importance (Supplementary File 2). The top 3 best features were an isomiR of hsa-miR-486-5p (iso23-8YUYFYKSY), piR-hsa-28723 and INTS10 for all histologies; Y-RNA, piR-hsa-28723 and GPB3 for NSCLC; and tRF-BS68BFD2, RN7SL724P and tRF-947673FE5 for SCLC. An in-depth investigation of selected features by other algorithms also showed common RNAs. For example, Y-RNA and iso-238YUYFYKSY isomiR were among the top predictors of the RF, elastic-net, the SGL and the lasso models for NSCLC; tRF-BS68BFD2 for SCLC. We also performed KEGG pathway enrichment analysis based on the common miRNA, mRNA and isomiR features. The results showed that many cancer-related pathways were significantly $(p<0.01)$ enriched such as MAPK signaling, mTOR signaling and AMPK signaling. 
We evaluated the classification performance of the XGBoost algorithm by selecting one sample per patient rather than using all samples from the same individuals. Our results showed comparable performance in terms of AUCs for all models (Figure 2 - Figure supplement 2). The SCLC models performed slightly worse than the others. This discrepancy can be explained by the relatively small sample size of this group. Therefore, we decided to use all samples from the same individuals.

\section{MiscRNA- and miRNA-only models are more accurate than the others}

We produced XGBoost models that included only a single RNA class (e.g. miRNA, isomiR etc.) to further investigate important features/classes. This method showed that miscRNA-only and miRNAonly models achieved better classification performance than the other RNA classes regardless of histology and stage at diagnosis (Table 2). The best separators of these models included, hsa-miR99a-5p, hsa-miR-1908-5p, hsa-miR-3925-5p and Y-RNA related transcripts (i.e RNY1P5 and RNY4P30). When we took histology into account, miRNAs and isomiRs for NSCLC and miscRNAs for SCLC produced better models (Table 2). The most important features of histology-dependent models included hsa-miR-629-5p, hsa-miR-99a-5p, hsa-miR-486-5p isomiR (iso-23-8YUYFYKSY), hsa-miR151a-3p isomiR (iso-22-B0NKZK1JN) for NSCLC; 7SL RNA related transcripts and Vault-RNA for SCLC (Supplementary File 2).

\section{Feature selection improves model performance and reduces model complexity}

Single RNA class models also implied that feature selection can further improve model performances. Thus, we tested two feature selection methods. The results showed lasso feature selection improved AUC values and reduced complexity (Table 2). The most important features of lasso-selected models included hsa-miR-423-5p isomiR (iso-20-5KP25HFF), GBP3 and piR-hsa-28723 for all histologies; YRNA, hsa-miR-423-5p isomiR (iso-20-5KP25HFF) and LINC01362 for NSCLC; HIST1H4E, PTCH2 and tRF-R29P4P9L5HJVE for SCLC (Supplementary File 2). Moreover, univariate significant feature selection greatly reduced model complexity with an acceptable performance (Table 2). For example, SCLC models only included 11 RNAs. The most important features were GBP3, LINC01362 and hsamiR-30a-5p for all histologies; LINC01362, GBP3 and tRF-9MV47P596V for NSCLC; piR-hsa-7001 and tRF-7343RX6NMH3 for SCLC (Supplementary File 2). 
214 Table 2. Averages of AUCs, accuracies (acc), sensitivities (sn) and specificities (sp) of the XGBoost 215 algorithm models on test datasets when prediagnostic time was not included.

\begin{tabular}{|c|c|c|c|c|c|c|c|c|c|}
\hline \multicolumn{9}{|c|}{ Histologies of model } & \multirow[b]{3}{*}{$\begin{array}{c}\text { Av. } \% \\
\text { acc/sn/sp }\end{array}$} \\
\hline & \multicolumn{2}{|c|}{ All (including others) } & \multicolumn{3}{|c|}{ NSCLC } & \multicolumn{3}{|c|}{ SCLC } & \\
\hline $\begin{array}{l}\text { Features } \\
\text { included: }\end{array}$ & AUC & $\begin{array}{c}\text { Av. \# of } \\
\text { features }\end{array}$ & $\begin{array}{c}\text { Av. \% of } \\
\text { acc/sn/sp }\end{array}$ & AUC & $\begin{array}{l}\text { Av. \# of } \\
\text { features }\end{array}$ & $\begin{array}{c}\text { Av. } \% \\
\text { acc/sn/sp }\end{array}$ & AUC & $\begin{array}{l}\text { Av. \# of } \\
\text { features }\end{array}$ & \\
\hline All RNAs & $\begin{array}{c}0.71(95 \% \\
\mathrm{Cl}, 0.68- \\
0.73)\end{array}$ & 301 & $69 / 73 / 62$ & $\begin{array}{c}0.70(95 \% \\
\text { Cl, 0.65- } \\
0.75)\end{array}$ & 373 & $67 / 70 / 64$ & $\begin{array}{c}0.71(95 \% \\
\text { Cl, 0.68- } \\
0.74)\end{array}$ & 213 & $70 / 69 / 71$ \\
\hline $\begin{array}{l}\text { Lasso- } \\
\text { selected } \\
\text { features }\end{array}$ & $\begin{array}{c}0.78(95 \% \\
\text { Cl, 0.74- } \\
0.82)\end{array}$ & 149 & $73 / 75 / 71$ & $\begin{array}{c}0.78(95 \% \\
\mathrm{Cl}, 0.75- \\
0.82)\end{array}$ & 56 & 73/73/72 & $\begin{array}{c}0.74(95 \% \\
\text { Cl, } 0.69- \\
0.80)\end{array}$ & 58 & $72 / 61 / 83$ \\
\hline $\begin{array}{l}\text { Univariate } \\
\text { significant } \\
\text { features }\end{array}$ & $\begin{array}{c}0.70(95 \% \\
\text { Cl, 0.66- } \\
0.73)\end{array}$ & 76 & $67 / 75 / 58$ & $\begin{array}{c}0.69(95 \% \\
\text { Cl, 0.64- } \\
0.73)\end{array}$ & 51 & $67 / 71 / 64$ & $\begin{array}{c}0.70(95 \% \\
\text { Cl, 0.65- } \\
0.76)\end{array}$ & 11 & $68 / 69 / 68$ \\
\hline $\begin{array}{l}\text { miRNA } \\
\text { only }\end{array}$ & $\begin{array}{c}0.72(95 \% \\
\text { Cl, 0.68- } \\
0.76)\end{array}$ & 168 & $69 / 76 / 61$ & $\begin{array}{c}0.73(95 \% \\
\text { Cl, 0.70- } \\
0.75)\end{array}$ & 199 & $69 / 74 / 64$ & $\begin{array}{c}0.65(95 \% \\
\text { Cl, 0.62- } \\
0.69)\end{array}$ & 20 & $67 / 74 / 60$ \\
\hline isomiR only & $\begin{array}{c}0.70(95 \% \\
\text { Cl, 0.65- } \\
0.74)\end{array}$ & 204 & $67 / 68 / 67$ & $\begin{array}{c}0.73(95 \% \\
\mathrm{Cl}, 0.69- \\
0.77)\end{array}$ & 215 & $71 / 75 / 66$ & $\begin{array}{c}0.65(95 \% \\
\text { Cl, 0.60- } \\
0.70)\end{array}$ & 108 & $66 / 65 / 67$ \\
\hline tRF only & $\begin{array}{c}0.69(95 \% \\
\text { Cl, 0.65- } \\
0.73)\end{array}$ & 314 & $65 / 77 / 53$ & $\begin{array}{c}0.67(95 \% \\
\text { Cl, 0.65- } \\
0.69)\end{array}$ & 314 & $66 / 64 / 67$ & $\begin{array}{c}0.68(95 \% \\
\text { Cl, 0.65- } \\
0.71)\end{array}$ & 23 & $66 / 69 / 63$ \\
\hline $\begin{array}{l}\text { miscRNA } \\
\text { only }\end{array}$ & $\begin{array}{c}0.72(95 \% \\
\text { Cl, 0.69- } \\
0.74)\end{array}$ & 83 & $69 / 73 / 65$ & $\begin{array}{c}0.68(95 \% \\
\text { Cl, 0.63- } \\
0.74)\end{array}$ & 87 & $66 / 73 / 59$ & $\begin{array}{c}0.69(95 \% \\
\text { Cl, 0.64- } \\
0.75)\end{array}$ & 76 & $70 / 78 / 61$ \\
\hline
\end{tabular}

${ }^{*}$ Average number of non-zero features selected by the models. Note: Detailed information on all selected features are in Supplementary File 2.

\section{Histology-specific prediagnostic models can improve prediction performance}

We previously demonstrated that RNA levels are dynamic and histology-specific in prediagnostic samples (Umu et al., 2019). We therefore trained and tested models stratified by prediagnostic time which were selected by a sliding window approach as explained in materials and methods.

The results showed inclusion of prediagnostic time and histological subtype together creates better models for specific time intervals (Figure 3). For example, SCLC models restricted to samples from 2 to 5 years prior to diagnosis had an average AUC of $0.84(95 \% \mathrm{Cl}, 0.77-0.9)$ (Figure 3). Another model of SCLC samples that only utilized miRNAs restricted to 3 to 5 years prior to diagnosis had an average AUC of $0.85(95 \% \mathrm{Cl}, 0.76-0.93)$ on the test datasets. Both SCLC models selected the same miRNAs as their most important features such as hsa-miR-30a-5p, hsa-miR-339-3p, hsa-miR-215-5p. 
Besides miRNAs, an isomiR of hsa-miR-451a and RN7SL181P were the most important features of prediagnostic SCLC models. Enrichment analysis of the most important features identified signaling pathways, such as MAPK, PI3K-Akt, RAS, and other pathways like choline metabolism, cellular senescence and PD-L1 expression \& PD-1 checkpoint. Similarly, NSCLC models restricted to 6 to 8 years prior to diagnosis had an average AUC of $0.81(95 \% \mathrm{Cl}, 0.75-0.86)$. The most important RNAs of this period were tRF-YP9L0N4V3, an isomiR of hsa-miR-484 (iso-23-8K4P8R8SDE) and tRF9MV47P596V. More than 70 pathways were enriched such as endocytosis, MAPK, RAS, choline metabolism and neurotrophin signaling pathway.

As an alternative to sliding windows, we also performed a fixed window approach and trained models using samples from up to 2 years (0-2), up to 5 years (0-5) and up to 8 years (0-8) before diagnosis. The results showed slight improvement in model performance compared to full time models (see Supplementary File 3). However, sliding windows models performed better on specific time intervals.

\section{Frequent features can create simple and accurate models}

We created models by compiling the best features from the full-time models. Our results showed improved prediction performance for these models despite inclusion of leave-out datasets into the test set (see materials and methods). In the test datasets including only smokers, AUC for all histologies was 0.76 (95\% Cl, 0.68-0.83); NSCLC model was 0.78 (95\% Cl, 0.70-0.85); SCLC model was 0.88 (95\% Cl, 0.83-0.94) (Table 3). However, when non-smokers were also included in the test set the model performance dropped to 0.68 (0.63-0.72) for all histologies and 0.68 (0.63-0.73) for NSCLC. Remarkably, the SCLC model still had AUC of 0.84 (0.79-0.9) when including non-smokers.

Table 3. All selected features, performance and RR of XGBoost models. 263

The RRs and their associated $p$ values on the test dataset, with and without non-smokers, are reported in Table 3. A positive test in smokers suggests more than two times higher risk of getting lung cancer diagnosis in future.

\begin{tabular}{|l|l|l|l|}
\hline & \multicolumn{3}{|c|}{ Models } \\
\hline & All $^{*}$ & NSCLC & SCLC \\
\hline
\end{tabular}




\begin{tabular}{|c|c|c|c|}
\hline Features & $\begin{array}{l}\text { iso-20-5KP25HFF } \\
\text { GBP3 } \\
\text { hsa-miR-30a-5p } \\
\text { INTS10 } \\
\text { LINC01362 } \\
\text { piR-hsa-28723 } \\
\text { RNU1-8P } \\
\text { iso-23-BQ8DQWM4Z } \\
\text { CTD-3252C9.4 } \\
\text { DST } \\
\text { HBA2 } \\
\text { HIST2H2AC } \\
\text { hsa-miR-99b-3p } \\
\text { LATS1 } \\
\text { piR-hsa-28391 } \\
\text { piR-hsa-28394 } \\
\text { RN7SL181P } \\
\text { RN7SL8P } \\
\text { RNU2-27P } \\
\text { iso-23-8YUYFYKSY } \\
\text { TLN1 } \\
\text { tRF-V47P59D9 } \\
\text { tRF-86V8WPMN1EJ3 } \\
\text { tRF-6SXMSL73VL4Y } \\
\text { tRF-QKF1R3WE8RO8IS }\end{array}$ & $\begin{array}{l}\text { LINC01362 } \\
\text { Y-RNA } \\
\text { iso-23-B0NKZ01J0D } \\
\text { iso-22-MKJIJLJ2Q } \\
\text { iso-21-N2NBQRZ00 } \\
\text { GBP3 } \\
\text { iso-20-RNUW92OI } \\
\text { GNAS } \\
\text { hsa-miR-30a-3p } \\
\text { NHSL2 } \\
\text { piR-hsa-28488 } \\
\text { RC3H2 } \\
\text { RN7SL181P } \\
\text { RNU2-19P } \\
\text { RNY4P27 } \\
\text { iso-23-909U247N04 } \\
\text { tRF-I89NJ4S2 } \\
\text { tRF-9MV47P596VE } \\
\text { tRF-86J8WPMN1EJ3 } \\
\text { tRF-86V8WPMN1EJ3 } \\
\text { tRF-Q1Q89P9L8422E }\end{array}$ & $\begin{array}{l}\text { AC113404.1 } \\
\text { C6orf223 } \\
\text { HIST1H4E } \\
\text { hsa-miR-30a-5p } \\
\text { hsa-miR-574-5p } \\
\text { ODC1 } \\
\text { PTCH2 } \\
\text { PTMA } \\
\text { RN7SL181P } \\
\text { tRF-22-947673FE5 } \\
\text { AKAP9 } \\
\text { MIGA1 } \\
\text { RAP1B } \\
\text { RN7SL724P } \\
\text { RUFY2 } \\
\text { iso-23-X3749W540L } \\
\text { tRF-BS68BFD2 } \\
\text { tRF-R29P4P9L5HJVE } \\
\text { tRF-ZRS3S3RX8HYVD }\end{array}$ \\
\hline Total features & 25 & 21 & 19 \\
\hline $\begin{array}{l}\text { Total test samples (total leave-out } \\
\text { size) (non-smokers) }\end{array}$ & $640(535)(263)$ & $465(360)(262)$ & $444(395)(256)$ \\
\hline $\begin{array}{l}\text { AUC on test }(95 \% \mathrm{Cl}) \\
\left.\text { (only smokers }{ }^{\star *}\right)\end{array}$ & $0.76(0.68-0.83)$ & $0.78(0.70-0.85)$ & $0.88(0.83-0.94)$ \\
\hline $\begin{array}{l}\text { AUC on test }(95 \% \mathrm{Cl}) \\
\left.\text { (both smokers and non-smokers }{ }^{\star *}\right)\end{array}$ & $0.68(0.63-0.72)$ & $0.68(0.63-0.73)$ & $0.84(0.79-0.9)$ \\
\hline $\begin{array}{l}\text { RR on test }(95 \% \mathrm{Cl}) \\
\left.\text { (only smokers }{ }^{\star \star}\right)\end{array}$ & $2.37(1.54-3.7) \mathrm{p}=1.15 \times 10^{-7}$ & $2.36(1.52-3.66) \mathrm{p}=2.83 \times 10^{-6}$ & $2.48(2.06-3) p=3.32 \times 10^{-9}$ \\
\hline $\begin{array}{l}\text { RR on test }(95 \% \mathrm{Cl}) \\
\left.\text { (both smokers and non-smokers }{ }^{\star *}\right)\end{array}$ & $1.84(1.7-2.01) \mathrm{p}=1.25 \times 10^{-6}$ & $1.52(1.27-1.83) \mathrm{p}=2.67 \times 10^{-5}$ & $2.04(1.85-2.25) \mathrm{p}=8.8 \times 10^{-8}$ \\
\hline
\end{tabular}

${ }^{*}$ Including other histologies. ${ }^{* *}$ includes samples previously not used (leave-out samples).

266 We also investigated the prediagnostic models, using the ML workflow, and selected two pairs of 267 models for NSCLC and SCLC, which showed high performance before and after 5 years prior to 268 diagnosis (see Supplementary File 4). We found that NSCLC models restricted to 0-2 years and 6-8 years before diagnosis had an average AUCs of $0.89(95 \% \mathrm{Cl}, 0.84-0.96)$ and $0.82(95 \% \mathrm{Cl}, 0.76$ 0.88) respectively; SCLC models restricted to 2-5 years and 8-10 years before diagnosis had an average AUCs of $0.89(95 \% \mathrm{Cl}, 0.77-1.0)$ and $0.83(95 \%, 0.69-0.97)$ respectively. We reported other model metrics and the best features in the supplementary document (Supplementary File 4).

\section{Discussion}

277 In this study, we showed that ML models of prediagnostic serum RNA levels can be used to predict LC 278 years before diagnosis and manifestation of disease symptoms. Our models achieved clinically 279 relevant performance in terms of AUC, accuracy, sensitivity and specificity (Table 2 and 3 ). The model 
performance was further increased for specific prediagnostic time windows and histologies making it feasible to develop them as biomarkers for LC screening (Figure 3). A collection of the best models (and predictors) (Table 3 and Supplementary File 4) can predict risk for developing LC, which histologies to look for and indicate the level of cancer progression. The time window of the highperformance models may be a first indication of how often to screen for LC (Figure 4). Our study is unique in including serum samples collected up to 10 years prior to LC diagnosis and a large set of control samples.

We previously reported prediagnostic circulating RNA signals are highly dynamic in lung cancer patients and they can be histology and stage dependent (Umu et al., 2019). In the present study, ML models using all samples regardless of stage, histology or prediagnostic time successfully separated LC patients from controls. All the tested algorithms consistently produced acceptable AUC values (Figure 2A). The best algorithm, XGBoost, resulted in an average accuracy of $69 \%$ without feature selection. An analysis of the features showed a large panel of selected RNAs: more than 300 out of available 3306 (with no feature selection implemented). This may be interpreted as a general shift in the levels of RNAs during cancer development, consistent with our previous study that showed hundreds of RNAs were differentially expressed up to 10 years before diagnosis (Umu et al., 2019).

We found that some features were considerably more important (and frequent) separators than others with or without feature selection. The list includes piRNAs (e.g. piR-hsa-28723), miRNAs (e.g. hsamiR-574-5p, hsa-miR-30a-5p, hsa-miR-106b-5p), isomiRs (e.g. isomiR of hsa-miR-423-5p (iso-205KP25HFF), hsa-miR-486-5p (iso-23-8YUYFYKSY)) and miscRNAs (e.g. RN7SL181P). Some of them were particularly interesting since they were associated with cancer or proposed as cancer biomarkers. Hsa-miR-30a-5p is a tumor suppressor and downregulated in LC tissues (Yanaihara et al., 2006). It regulates oncogenes such as RAB38 and RAB27B (The RNAcentral Consortium, 2019). Another notable example is hsa-miR-574-5p which promotes metastasis in NSCLC by targeting PCP2 in tumor tissues (Zhou et al., 2016) and has been proposed as an early stage NSCLC serum biomarker (Foss et al., 2011). Hsa-miR-574-5p was among the most important features in lassoselected and miRNA-only histology-specific SCLC models. It was also one of the most important 309 features in our histology-specific NSCLC models. There were also isomiRs among the most important features such as hsa-miR-486-5p canonical form, which was the best separator for all histologies. Hsa-miR-486-5p targets PIK3R1 to suppress cell growth. Its overexpression inhibits cell proliferation and invasion and it was significantly downregulated in both tissue and serum (Tian et al., 2019). HsamiR-486-5p was proposed as a diagnostic and prognostic biomarker for NSCLC (ElKhouly et al., 2020; Tian et al., 2019). 
Besides miRNAs and isomiRs, RNAs of other classes were noteworthy and linked to carcinogenesis. For example, 7SL, a member of miscRNAs, is upregulated in tumor cells. It binds to TP53 mRNA at the 3'UTR region and downregulates its expression (Abdelmohsen et al., 2014). 7SL related transcripts (e.g. RN7SL181P) were among the most important separators in the all histology, NSCLCand SCLC-specific models. Another example is Y-RNA and we found that Y-RNA and related genes (e.g. RNY4P30) were among the most important features for NSCLC models. Y-RNA was also chosen 322 as an important feature by the lasso-selected NSCLC models. Y-RNA derived small RNAs function as tumor suppressors in NSCLC. They inhibit cell proliferation and were proposed as circulating RNA biomarkers since they were upregulated in NSCLC EVs (Li et al., 2018).

Inclusion of both prediagnostic time and histology produced better models in certain time windows (e.g. 2 to 5 years before diagnosis for SCLC) (Figure 3). This can be explained by the dynamic nature 328 of prediagnostic RNA levels (Lund et al., 2016; Umu et al., 2019). Important features of these models can also be linked to early carcinogenesis and some were specific to these models. For example, hsamiR-339-3p was among the most important features of SCLC prediagnostic models. Hsa-miR-339-3p is a tumor suppressor and was proposed as a serum biomarker of LC (Yu et al., 2019). We retrained some of these prediagnostic models using the most frequent features and achieved higher prediction performance than the full-time models in specific time intervals. We reported these models in supplementary (Supplementary File 3).

The most important features of histology-specific models also showed associations with carcinogenesis related KEGG pathways, which were common or specific to histology. The common ones include well-known signaling (e.g. RAS, PI3K-Akt, MAPK, ErbB) and cancer-related pathways (e.g. proteoglycans in cancer and pathways in cancer). Choline metabolism in cancer pathway was one of the common ones and enriched in some histology-specific prediagnostic models. Altered choline profiles are characteristics of tumor tissues (Glunde et al., 2006). Moreover, a lipidome serum profiling study on early stage NSCLC patients proposed choline-containing phospholipids as potential LC biomarkers (Klupczynska et al., 2019). Enrichment of choline metabolism pathway years before diagnosis (i.e. NSCLC 6-8 and SCLC 2-5) supports this conclusion. We also reported enrichment of this pathway for all histologies before diagnosis in our previous study (Umu et al., 2019).

A strength of our study is the large sample size from prediagnostic cases and a large control group from cancer-free individuals from the same cohort. We have detailed information on histological subtype and stage at diagnosis from the Cancer Registry of Norway and smoking history from survey data. We also accounted for other potential confounders (i.e. age, sex and BDg) (Rounge et al., 2018). 
associated with carcinogenesis or proposed as biomarkers, which shows consistent results with current literature. Further, we found potential biomarkers from overlooked RNA classes which add important new knowledge into the field. We shared the average feature importance of all RNAs as supplementary tables (Supplementary File 2). We investigated performance of different algorithms which showed consistent results in terms of AUCs and features. We compiled shortlists from the most important features and tested their performance in a leave-out dataset on both smokers and nonsmokers. We also found that smokers with a positive test had more than two times higher risk of getting lung cancer diagnosis in future (Table 3 ).

There are some weaknesses in our study that we need to address. First, an independent cohort should replicate our results. However, only a few cohorts include prediagnostic samples that can be used for discovery and validation. We tried to overcome this issue by using training-testing repeats for assessing generalisability. We also reported our results with and without feature selection since some feature selection methods (e.g. lasso and univariate) can cause overfitting. Second, using more than one sample from the same individual can potentially cause overfitting. However, we did not detect any effect related to this issue (Figure 2 - Figure supplement 2). Third, our study focused only on smokers (since case samples are mostly smokers). However, our results show acceptable performance when including non-smokers as a test dataset as well. Fourth, reuse of the same data for frequent biomarker models (as reported in Table 3) can also result in overfitting. We tried to overcome this issue by including a leave-out dataset (which was never used) into the test set and reported performance. Lastly, since our samples are long-term stored, some unstable RNA molecules may have been degraded over the years, though we have already shown that this effect is negligible (Umu et al., 2018). Yet, we matched cases and controls for BDg which includes the effect of storage time (see materials and methods).

In LC screening programs, RNA biomarkers can be used as a tool of initial assessment or combined with LDCT for early detection (Hanash et al., 2018). We found that smokers with a positive test had higher risk of getting lung cancer diagnosis in future (Table 3). We also found that our biomarkers can be potentially used on non-smokers, especially SCLC biomarkers. However, we do not have enough non-smoker cases to further validate this interpretation. The dynamic nature of the prediagnostic signal for cancer may pose challenges for the performance of modeling and biomarker development. However, using a set of models specific for histology and time might provide additional information useful in evaluating LC risk (Figure 4). Our proposed use of RNA biomarkers starts with risk assessment using standard full-time models which can be used for an initial assessment in smokers 
when the disease is undetectable. A positive signal (i.e. high probability of being in LC group) classifies those individuals into an elevated risk group. Since prediagnostic models have a two-year peak performance, every second-year testing with these models can provide confirmation of preneoplasia or an early stage tumor for individuals with elevated risk and selection criteria for CT monitoring. Prediagnostic models had higher overall specificity (more than $80 \%$ ) which can help to determine future diagnosis histology. However, it requires further research. We selected two sets of histology-specific diagnostic models for early/late NSCLC and SCLC diagnosis and reported these in the supplementary document (Supplementary File 4). RNA biomarkers can prevent unnecessary use of LDCT while improving the chance of an early diagnosis of LC in an early stage. This hypothesis can be investigated in screening programs for validation.

\section{Conclusion}

We have shown that LC can be detected in both smokers and non-smokers years before diagnosis and the manifestation of symptoms regardless of histological subtype. We also proposed a model on how RNA biomarkers can be utilized in clinical settings. Our top performing models can produce AUCs up to 0.9 before diagnosis suggesting a great potential for LC early prediction.

\section{Acknowledgements}

This work was supported by The Norwegian Research Council's Programme 'Human Biobanks and Health Data [229621/H10, 248791/H10]. Disclosure of invention was accepted by the technology transfer office, Invent2 (DOFI: 19010). We would like to acknowledge Cecilie Bucher-Johannessen, Marianne Lauritzen, Magnus Leithaug for performing lab and coordination tasks. We also acknowledge Matthew D. Whitaker and Marc Chadeau-Hyam from Imperial College London for discussions on ML model training and testing. We acknowledge the Norwegian Institute of Public Health for access to survey data in this study. The sequencing service was provided by the Norwegian Sequencing Centre (www.sequencing.uio.no), a national technology platform hosted by Oslo University Hospital and the University of Oslo supported by the Research Council of Norway and the Southeastern Regional Health Authority.

\section{Materials and Methods}

\section{Study population and data sources}

We used the population based Janus Serum Bank (JSB) cohort containing prediagnostic serum samples (Langseth et al., 2017). The study participants were identified by linking the JSB to the Cancer Registry of Norway (CRN). We restricted our analyses to patients later diagnosed with LC up to 10 years after blood donation and control samples from individuals cancer-free (except non- 
melanoma skin cancer) at least 10 years after sample collection. We matched cases and controls on confounders (see bioinformatics analyses). Smoking, collected from health survey data, was classified as current, former, or never smokers (Hjerkind et al., 2017). Since we have previously shown that smoking significantly affects RNA expression levels, we only included smokers (ie. current and former) in the initial analyses and model building. However, non-smokers and samples not included by the frequency matching were used as an additional independent leave-out dataset to assess the level of overfitting.

\section{Tumor staging}

Detailed cancer information was selected from the CRN that has systematically collected mandatory notification on cancer occurrence for the Norwegian population since 1952 (Larsen et al., 2009). The cases were classified into histological subtypes: NSCLC, SCLC and others, the latter referring to other less defined or multiple histologies. Stage at diagnosis was encoded with the TNM system: early (localized - stage I), locally advanced (regional - stage II and III), advanced or metastatic (distant stage IV) and unknown (Cancer Registry of Norway, 2020).

\section{Laboratory processing}

We extracted RNA from $400 \mu \mathrm{L}$ serum using phenol-chloroform and miRNeasy Serum/Plasma kit (Qiagen, Valencia, CA, USA). We performed size selection using a 3\% Agarose Gel Cassette (Cat. No CSD3010) on a Pippin Prep (Sage Science) with a cut size optimized to cover RNA molecules from 17 to $47 \mathrm{nt}$ in length. Libraries were prepared with the NEBNext Small RNA kit (NEB, Ipswich, MA, USA) and sequenced on a HiSeq 2500 platform to on average 18 million sequences per sample (Illumina, San Diego, CA, USA).

\section{Bioinformatic analyses}

Our bioinformatics workflow includes quality control, adapter trimming, read mapping, read counting and creation of count tables. We used a large annotation dataset containing several RNA classes available in serum (Umu et al., 2018), including miRBase (v22.1) for miRNAs (Kozomara et al., 2019), piRBase (v1.0) for piRNAs (Zhang et al., 2014) and the GENCODE (v26) for other RNA classes (Harrow et al., 2012). We used the AdapterRemoval tool for adapter trimming (Schubert et al., 2016) and Bowtie2 (Langmead and Salzberg, 2012) for mapping reads to the human genome (hg38) with an average mapping ratio of $70 \%$. The SeqBuster tool was used for miRNA annotation counts and isomiR calling (Pantano et al., 2010). We filtered out the RNAs with fewer than 5 reads in less than $80 \%$ of the samples. All isomiRs passed the expression were regarded as bona fide isomiRs. We used DESeq2's (Love et al., 2014) variance stabilizing normalization function to normalize identified RNA counts. The optmatch (v0.9-11) R package (Hansen and Klopfer, 2006) selected appropriately matched controls 
while building models. Therefore, we matched LC samples and controls on sex, age at donation, and blood donor group (BDg). BDg is a technical cofounder combining the effect of sample treatment at donation and storage time (Rounge et al., 2018). We used $R$ function kegga from the limma package for KEGG pathway enrichment analysis of selected RNA features if they are miRNA, isomiR or mRNA. The miRNA and isomiR targets were extracted from MIRDB (v5.0) predictions (Wong and Wang, 2015) (score cutoff $>60$ ). $P$ values were adjusted using false discovery rate (FDR) (using p.adjust function of $R$ ).

\section{Machine learning classification algorithms and training/testing workflow}

High dimensionality is often a problem in modelling RNA-seq data. Our preliminary analysis showed that $\mathrm{ML}$ algorithms with regularization produced successful models. Therefore, we selected five ML algorithms to create our initial models: lasso, elastic-net, sparse group lasso (SGL), random forest (RF) and extreme gradient boosting (XGBoost) algorithms. We used 5-fold cross-validation (if available) to tune hyperparameters for model training. For the SGL models, RNAs were classified by type.

$\mathrm{R}$ implementations of these algorithms were used: caret (v6.0-84) and glmnet (v2.0-18) packages for elastic-net and the lasso, sglfast (v0.10) and msgl (v2.3.9) for the SGL models and xgboost (v1.0.0.2) for XGBoost. Classifications were performed according to histology and time to diagnosis (for details see next paragraph) using an automated ML workflow. In the ML workflow the datasets were split into training $(70 \%)$ and test $(30 \%)$ (Figure 1B). We repeated this step 5 times using designated seed numbers to select 5 different training and test datasets which were balanced for case/control numbers and also matched for confounders (i.e sex, age and BDg). Model optimization including hyperparameter tuning was done by a grid search approach followed by 5 -fold cross-validation using the training sets. The test datasets were only used for testing to overcome overfitting and assess true performance. The performance of the classifiers were mainly evaluated by area under the ROC curves (AUC)s. We also calculated accuracy, sensitivity and specificity. Confidence interval calculations were done using metrics of test datasets.

\section{Histology and prediagnostic models}

We refer to models for all histologies, NSCLC and SCLC that do not take time to diagnose into account as standard full-time models (Figure 1B). Prediagnostic models were created using a sliding windows approach and a fixed-time approach to find optimal time to diagnose intervals. We first selected 3 different window sizes, 2, 3 and 4 years, which were moved over the 10 years prior to diagnosis time. We then built models based on samples captured by these sliding windows. Fixed time 
windows were $0-2,0-5$ and $0-8$ years before diagnosis. We used the workflow described above to train and test both standard and prediagnostic models.

\section{Feature selection methods}

We implemented feature selection methods to improve model performances, including single-RNA class, lasso-selection and significant-selection. In the single-RNA class method, we dropped all RNA types except one. In lasso-selection, all non-zero features selected by the lasso classification models were pooled. Next, we retrained new classification models which were restricted to use only these features. In significance-selection, an univariate regression analysis was done per feature and significant features (multiple testing adjusted) were used to train classification models.

\section{Frequent feature models, independent leave-out test and relative risk (RR) calculations} We created models for each histology which utilize the most frequent features identified in the standard full-time models. To assess overfitting and to get a better estimate of these model performances, we split the datasets into training $(80 \%)$ and test $(20 \%)$ sets. To the test sets we also added non-smokers and samples from smokers, but not previously used in frequency matching (number of samples reported in Table 3). Both unmatchable samples and non-smokers were never used for model building and evaluation which we refer to as leave-out sets. We did not repeat this analysis 5 times as in the automated ML workflow. RRs were calculated using the test sets. The optimal threshold was identified in cross-validation. We used the R packages cutpointr (v1.0.1) and epitools (v0.5-10.1) to calculate RRs.

\section{Ethics}

This study was approved by the Norwegian Regional Committee for medical and health research ethics (REC no: 19892 previous 2016/1290) and was based on a broad consent from participants in the Janus cohort. The work has been carried out in compliance with the standards set by the Declaration of Helsinki.

\section{Data Accessibility}

The datasets generated for this article are not readily available because of the principles and conditions set out in articles 6 (1) (e) and 9 (2) (j) of the General Data Protection Regulation (GDPR). National legal basis as per the Regulations on population-based health surveys and ethical approval from the Norwegian Regional Committee for Medical and Health Research Ethics (REC) is also required. Requests to access the datasets should be directed to the corresponding authors. Our scripts and bioinformatics workflow files can be accessed from our Github repo (https://github.com/sinanugur/LCscripts). 
Abdelmohsen K, Panda AC, Kang M-J, Guo R, Kim J, Grammatikakis I, Yoon J-H, Dudekula DB, Noh $\mathrm{JH}$, Yang X, Martindale JL, Gorospe M. 2014. 7SL RNA represses p53 translation by competing with HuR. Nucleic Acids Res 42:10099-10111.

Aberle DR, Berg CD, Black WC, Church TR, Fagerstrom RM, Galen B, Gareen IF, Gatsonis C, Goldin J, Gohagan JK, Hillman B, Jaffe C, Kramer BS, Lynch D, Marcus PM, Schnall M, Sullivan DC, Sullivan D, Zylak CJ. 2011. The National Lung Screening Trial: overview and study design. Radiology 258:243-253.

Bach PB, Mirkin JN, Oliver TK, Azzoli CG, Berry DA, Brawley OW, Byers T, Colditz GA, Gould MK, Jett JR, Sabichi AL, Smith-Bindman R, Wood DE, Qaseem A, Detterbeck FC. 2012. Benefits and harms of CT screening for lung cancer: a systematic review. JAMA 307:2418-2429.

Bray F, Ferlay J, Soerjomataram I, Siegel RL, Torre LA, Jemal A. 2018. Global cancer statistics 2018: GLOBOCAN estimates of incidence and mortality worldwide for 36 cancers in 185 countries. CA Cancer J Clin 68:394-424.

Brustugun OT, Grønberg BH, Fjellbirkeland L, Helbekkmo N, Aanerud M, Grimsrud TK, Helland Å, Møller B, Nilssen Y, Strand TE, Solberg SK. 2018. Substantial nation-wide improvement in lung cancer relative survival in Norway from 2000 to 2016. Lung Cancer 122:138-145.

Burton J, Umu SU, Langseth H, Grotmol T, Grimsrud TK, Haugen TB, Rounge TB. 2020. Serum RNA Profiling in the 10-Years Period Prior to Diagnosis of Testicular Germ Cell Tumor. doi:10.3389/fonc.2020.574977

Cancer Registry of Norway. 2020. Cancer in Norway 2019 - Cancer incidence, mortality, survival and prevalence in Norway.

de Koning HJ, van der Aalst CM, de Jong PA, Scholten ET, Nackaerts K, Heuvelmans MA, Lammers J-WJ, Weenink C, Yousaf-Khan U, Horeweg N, van 't Westeinde S, Prokop M, Mali WP, Mohamed Hoesein FAA, van Ooijen PMA, Aerts JGJV, den Bakker MA, Thunnissen E, Verschakelen J, Vliegenthart R, Walter JE, Ten Haaf K, Groen HJM, Oudkerk M. 2020. Reduced Lung-Cancer Mortality with Volume CT Screening in a Randomized Trial. N Engl J Med 382:503513.

EIKhouly AM, Youness RA, Gad MZ. 2020. MicroRNA-486-5p and microRNA-486-3p: Multifaceted pleiotropic mediators in oncological and non-oncological conditions. Noncoding RNA Res 5:1121.

Fehlmann T, Kahraman M, Ludwig N, Backes C, Galata V, Keller V, Geffers L, Mercaldo N, Hornung D, Weis T, Kayvanpour E, Abu-Halima M, Deuschle C, Schulte C, Suenkel U, von Thaler A-K, Maetzler W, Herr C, Fähndrich S, Vogelmeier C, Guimaraes P, Hecksteden A, Meyer T, Metzger F, Diener C, Deutscher S, Abdul-Khaliq H, Stehle I, Haeusler S, Meiser A, Groesdonk HV, Volk T, Lenhof H-P, Katus H, Balling R, Meder B, Kruger R, Huwer H, Bals R, Meese E, Keller A. 2020. Evaluating the Use of Circulating MicroRNA Profiles for Lung Cancer Detection in Symptomatic Patients. JAMA Oncol 6:714-723.

Foss KM, Sima C, Ugolini D, Neri M, Allen KE, Weiss GJ. 2011. miR-1254 and miR-574-5p: serumbased microRNA biomarkers for early-stage non-small cell lung cancer. $J$ Thorac Oncol 6:482488.

Freedman JE, Gerstein M, Mick E, Rozowsky J, Levy D, Kitchen R, Das S, Shah R, Danielson K, Beaulieu L, Navarro FCP, Wang Y, Galeev TR, Holman A, Kwong RY, Murthy V, Tanriverdi SE, Koupenova-Zamor M, Mikhalev E, Tanriverdi K. 2016. Diverse human extracellular RNAs are widely detected in human plasma. Nat Commun 7:11106.

Fritz JV, Heintz-Buschart A, Ghosal A, Wampach L, Etheridge A, Galas D, Wilmes P. 2016. Sources and Functions of Extracellular Small RNAs in Human Circulation. Annu Rev Nutr 36:301-336. 
Glunde K, Jacobs MA, Bhujwalla ZM. 2006. Choline metabolism in cancer: implications for diagnosis and therapy. Expert Rev Mol Diagn 6:821-829.

Gopal M, Abdullah SE, Grady JJ, Goodwin JS. 2010. Screening for lung cancer with low-dose computed tomography: a systematic review and meta-analysis of the baseline findings of randomized controlled trials. J Thorac Oncol 5:1233-1239.

Gutschner T, Diederichs S. 2012. The hallmarks of cancer: a long non-coding RNA point of view. RNA Biol 9:703-719.

Hanahan D, Weinberg RA. 2000. The hallmarks of cancer. Cell 100:57-70.

Hanash SM, Ostrin EJ, Fahrmann JF. 2018. Blood based biomarkers beyond genomics for lung cancer screening. Trans/ Lung Cancer Res 7:327-335.

Hansen BB, Klopfer SO. 2006. Optimal Full Matching and Related Designs via Network Flows. J Comput Graph Stat 15:609-627.

Harrow J, Frankish A, Gonzalez JM, Tapanari E, Diekhans M, Kokocinski F, Aken BL, Barrell D, Zadissa A, Searle S, Barnes I, Bignell A, Boychenko V, Hunt T, Kay M, Mukherjee G, Rajan J, Despacio-Reyes G, Saunders G, Steward C, Harte R, Lin M, Howald C, Tanzer A, Derrien T, Chrast J, Walters N, Balasubramanian S, Pei B, Tress M, Rodriguez JM, Ezkurdia I, van Baren J, Brent M, Haussler D, Kellis M, Valencia A, Reymond A, Gerstein M, Guigó R, Hubbard TJ. 2012. GENCODE: the reference human genome annotation for The ENCODE Project. Genome Res 22:1760-1774.

Hjerkind KV, Gislefoss RE, Tretli S, Nystad W, Bjørge T, Engeland A, Meyer HE, Holvik K, Ursin G, Langseth H. 2017. Cohort Profile Update: The Janus Serum Bank Cohort in Norway. Int J Epidemiol 46:1101-1102f.

Keller A, Meese E. 2016. Can circulating miRNAs live up to the promise of being minimal invasive biomarkers in clinical settings? Wiley Interdiscip Rev RNA 7:148-156.

Kim KM, Abdelmohsen K, Mustapic M, Kapogiannis D, Gorospe M. 2017. RNA in extracellular vesicles. Wiley Interdiscip Rev RNA 8. doi:10.1002/wrna.1413

Klupczynska A, Plewa S, Kasprzyk M, Dyszkiewicz W, Kokot ZJ, Matysiak J. 2019. Serum lipidome screening in patients with stage I non-small cell lung cancer. Clin Exp Med 19:505-513.

Ko J, Baldassano SN, Loh P-L, Kording K, Litt B, Issadore D. 2018. Machine learning to detect signatures of disease in liquid biopsies - a user's guide. Lab Chip 18:395-405.

Kozomara A, Birgaoanu M, Griffiths-Jones S. 2019. miRBase: from microRNA sequences to function. Nucleic Acids Res 47:D155-D162.

Langmead B, Salzberg SL. 2012. Fast gapped-read alignment with Bowtie 2. Nat Methods 9:357-359.

Langseth H, Gislefoss RE, Martinsen JI, Dillner J, Ursin G. 2017. Cohort Profile: The Janus Serum Bank Cohort in Norway. Int J Epidemiol 46:403-404g.

Larsen IK, Småstuen M, Johannesen TB, Langmark F, Parkin DM, Bray F, Møller B. 2009. Data quality at the Cancer Registry of Norway: an overview of comparability, completeness, validity and timeliness. Eur J Cancer 45:1218-1231.

Li C, Qin F, Hu F, Xu H, Sun G, Han G, Wang T, Guo M. 2018. Characterization and selective incorporation of small non-coding RNAs in non-small cell lung cancer extracellular vesicles. Cell Biosci 8:2.

Love MI, Huber W, Anders S. 2014. Moderated estimation of fold change and dispersion for RNA-seq data with DESeq2. Genome Biol 15:550.

Lund E, Holden L, Bøvelstad H, Plancade S, Mode N, Günther C-C, Nuel G, Thalabard J-C, Holden M. 2016. A new statistical method for curve group analysis of longitudinal gene expression data illustrated for breast cancer in the NOWAC postgenome cohort as a proof of principle. BMC Med Res Methodol 16:28.

Murillo OD, Thistlethwaite W, Rozowsky J, Subramanian SL, Lucero R, Shah N, Jackson AR, Srinivasan S, Chung A, Laurent CD, Kitchen RR, Galeev T, Warrell J, Diao JA, Welsh JA, Hanspers K, Riutta A, Burgstaller-Muehlbacher S, Shah RV, Yeri A, Jenkins LM, Ahsen ME, Cordon-Cardo C, Dogra N, Gifford SM, Smith JT, Stolovitzky G, Tewari AK, Wunsch BH, Yadav KK, Danielson KM, Filant J, Moeller C, Nejad P, Paul A, Simonson B, Wong DK, Zhang X, Balaj L, Gandhi R, Sood AK, Alexander RP, Wang L, Wu C, Wong DTW, Galas DJ, Van Keuren- 

Jensen K, Patel T, Jones JC, Das S, Cheung K-H, Pico AR, Su AI, Raffai RL, Laurent LC, Roth ME, Gerstein MB, Milosavljevic A. 2019. exRNA Atlas Analysis Reveals Distinct Extracellular RNA Cargo Types and Their Carriers Present across Human Biofluids. Cell 177:463-477.e15.

Pantano L, Estivill X, Martí E. 2010. SeqBuster, a bioinformatic tool for the processing and analysis of small RNAs datasets, reveals ubiquitous miRNA modifications in human embryonic cells. Nucleic Acids Res 38:e34.

Peled N, llouze M. 2015. Screening for Lung Cancer: What Comes Next? J Clin Oncol.

Pichler M, Calin GA. 2015. MicroRNAs in cancer: from developmental genes in worms to their clinical application in patients. Br J Cancer 113:569-573.

Rounge TB, Umu SU, Keller A, Meese E, Ursin G, Tretli S, Lyle R, Langseth H. 2018. Circulating small non-coding RNAs associated with age, sex, smoking, body mass and physical activity. Sci Rep 8:1760.

Sandfeld-Paulsen B, Jakobsen KR, Bæk R, Folkersen BH, Rasmussen TR, Meldgaard P, Varming K, Jørgensen MM, Sorensen BS. 2016. Exosomal Proteins as Diagnostic Biomarkers in Lung Cancer. J Thorac Oncol 11:1701-1710.

Schubert M, Lindgreen S, Orlando L. 2016. AdapterRemoval v2: rapid adapter trimming, identification, and read merging. BMC Res Notes 9:88.

Seijo LM, Peled N, Ajona D, Boeri M, Field JK, Sozzi G, Pio R, Zulueta JJ, Spira A, Massion PP, Mazzone PJ, Montuenga LM. 2019. Biomarkers in Lung Cancer Screening: Achievements, Promises, and Challenges. J Thorac Oncol 14:343-357.

Slack FJ, Chinnaiyan AM. 2019. The Role of Non-coding RNAs in Oncology. Cell 179:1033-1055.

Svoronos AA, Engelman DM, Slack FJ. 2016. OncomiR or Tumor Suppressor? The Duplicity of MicroRNAs in Cancer. Cancer Res 76:3666-3670.

The RNAcentral Consortium. 2019. RNAcentral: a hub of information for non-coding RNA sequences. Nucleic Acids Res 47:D1250-D1251.

Tian F, Wang J, Ouyang T, Lu N, Lu J, Shen Y, Bai Y, Xie X, Ge Q. 2019. MiR-486-5p Serves as a Good Biomarker in Nonsmall Cell Lung Cancer and Suppresses Cell Growth With the Involvement of a Target PIK3R1. Front Genet 10:688.

Umu SU, Langseth H, Bucher-Johannessen C, Fromm B, Keller A, Meese E, Lauritzen M, Leithaug M, Lyle R, Rounge TB. 2018. A comprehensive profile of circulating RNAs in human serum. RNA Biol 15:242-250.

Umu SU, Langseth H, Keller A, Meese E, Helland Å, Lyle R, Rounge TB. 2019. A 10 year prediagnostic followup study shows that serum RNA signals are highly dynamic in lung carcinogenesis. Mol Oncol.

Wild CP, Weiderpass W, Stewart BW. 2020. World Cancer Report: Cancer Research for Cancer Prevention. Lyon, France: International Agency for Research on Cancer.

Wong N, Wang X. 2015. miRDB: an online resource for microRNA target prediction and functional annotations. Nucleic Acids Res 43:D146-52.

Yanaihara N, Caplen N, Bowman E, Seike M, Kumamoto K, Yi M, Stephens RM, Okamoto A, Yokota J, Tanaka T, Calin GA, Liu C-G, Croce CM, Harris CC. 2006. Unique microRNA molecular profiles in lung cancer diagnosis and prognosis. Cancer Cell 9:189-198.

Yu H, Guan Z, Cuk K, Zhang Y, Brenner H. 2019. Circulating MicroRNA Biomarkers for Lung Cancer Detection in East Asian Populations. Cancers 11. doi:10.3390/cancers11030415

Zaporozhchenko IA, Ponomaryova AA, Rykova EY, Laktionov PP. 2018. The potential of circulating cell-free RNA as a cancer biomarker: challenges and opportunities. Expert Rev Mol Diagn 18:133-145.

Zhang P, Si X, Skogerbø G, Wang J, Cui D, Li Y, Sun X, Liu L, Sun B, Chen R, He S, Huang D-W. 2014. piRBase: a web resource assisting piRNA functional study. Database 2014:bau110.

Zhou R, Zhou X, Yin Z, Guo J, Hu T, Jiang S, Liu L, Dong X, Zhang S, Wu G. 2016. MicroRNA-574-5p promotes metastasis of non-small cell lung cancer by targeting PTPRU. Sci Rep 6:35714. 
Figure 1. A. The sample selection is summarized by the flow chart. Non-smokers were excluded from 691 model building. B. We randomly created five different training and testing datasets for each group (e.g. standard, histology-specific or prediagnostic models).

693 Figure 2. Each ROC curve is based on the prediction results of a randomly created testing dataset (in 694 695 total 5). AUC values show the average of these predictions. The most important features of the classifiers were sorted on their average feature importance and are shown in the lower panels. A 696 Detailed list of biomarkers with their feature importance is available in supplementary (Supplementary 697 698 699 700 701 702 703 704 705 706 File 2). We did not perform any feature selection while training these models. (see also Figure 2 Source Data)

Figure 2 - Figure supplement 1. Each boxplot shows performances of an algorithm measured by AUCs. The analyses were done for all histologies and histology-specific (i.e. separately for NSCLC and SCLC), regardless of prediagnostic time. The dashed lines represent the combined average performances of all tested algorithms. XGBoost produced an above average prediction performance. (Figure 2 - Source Data 1)

Figure 2 - Figure supplement 2. A The performance of the XGBoost algorithm with all samples. B. When one sample per individual was selected, the classification performance was comparable for all models. (Figure 2 - Source Data 2)

Figure 3. Sliding windows analysis showed better models which utilizes prediagnostic samples in specific time intervals such as SCLC models, which were restricted to samples from 2 to 5 years prior to diagnosis (see the first and the second panel, red dots). Each color represents different histologies: black and red only have NSCLC and SCLC samples respectively while blue has all histologies including others. (Figure 3 - Source Data)

Figure 4. Suggested clinical uses of RNA biomarkers in LC screening. A positive test from full-time models shows elevated risk (at least 2 times). They can detect cancer-related RNA signals up to 10 years before diagnosis. Prediagnostic models have higher accuracy, sensitivity and specificity which can potentially assist full-time models and improve specificity (Supplementary File 4).

Supplementary File 1. Clinical and histological characteristics of nonsmoker samples of leave-out 719 dataset.

720 Supplementary File 2. Detailed feature importance tables for all trained models. 
721 Supplementary File 3. Fixed-time model performance on different histologies.

722 Supplementary File 4. Selected prediagnostic models, metrics and their feature importance tables.

723

724 Figure 2 - Source data. Source data of XGBoost ROC plots for Figure 2.

725 Figure 2 - Source data 1. Source data of boxplots for Figure 2 - Figure supplement 1.

726 Figure 2 - Source data 2. Source data of ROC plots without multiple samples from same individuals

727 Figure 2 - Figure supplement 2.

728 Figure 3 - Source data. Source data of all the panels for Figure 3.

729

730

731

732

733

734 

ROC curves of XGBoost models

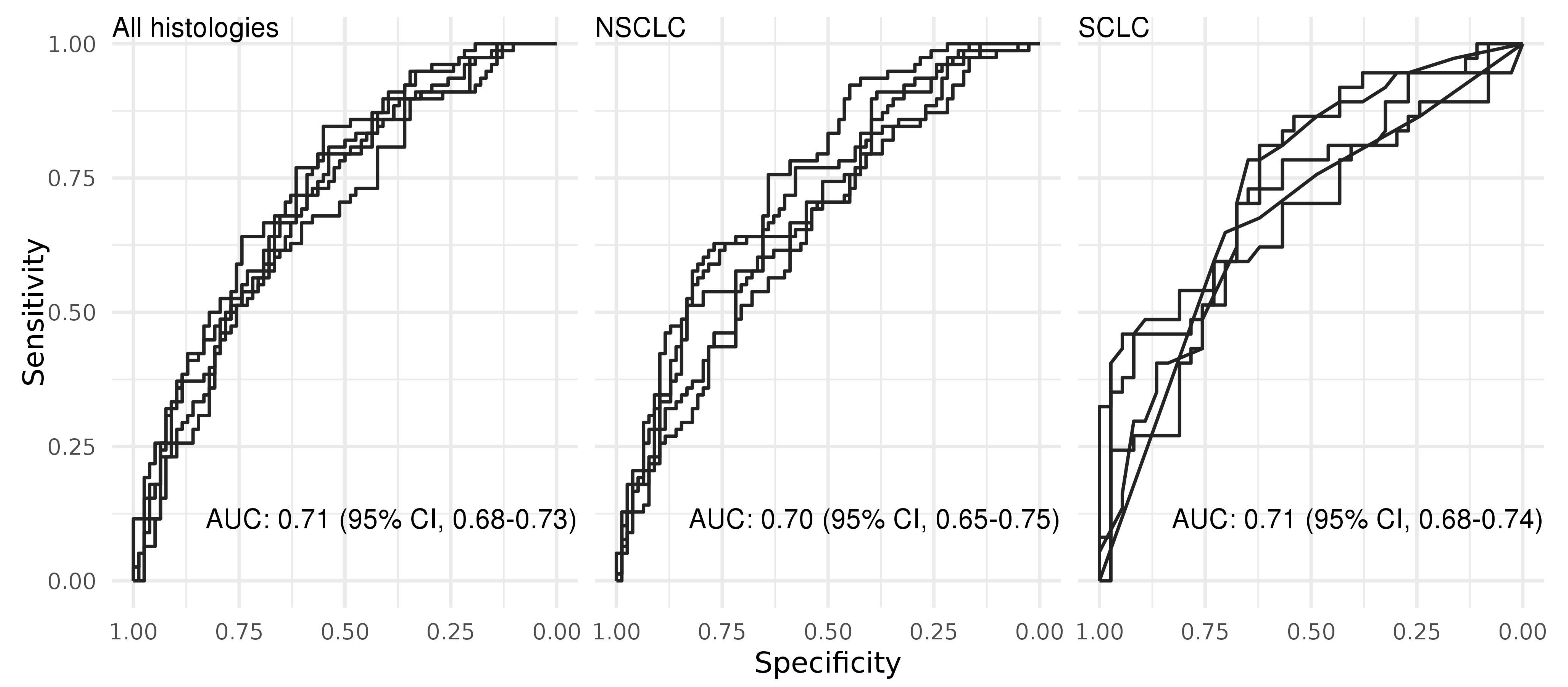

Feature importance

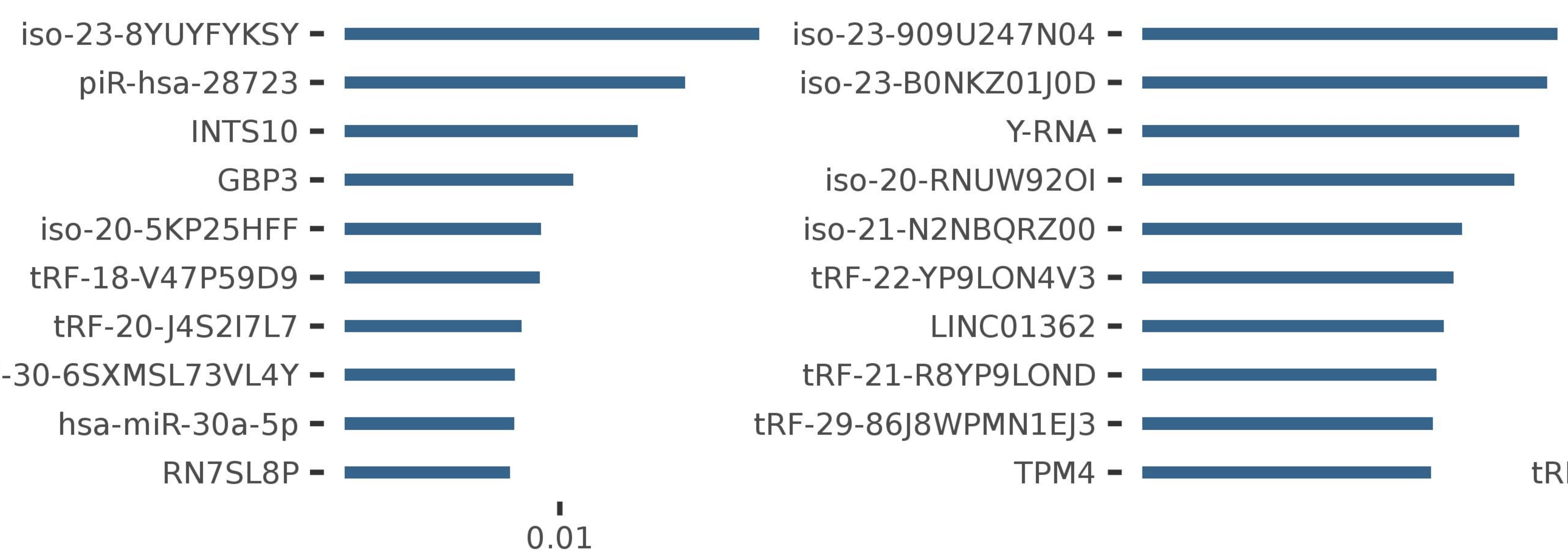

tRF-18-BS68BFD2 -

RN7SL724P -

tRF-22-947673FE5 -

$\mathrm{PTCH} 2$ -

PTMA -

RN7SL181P -

hsa-miR-574-5p -

AC113404.1 -

HIST1H4E -

RF-31-ZRS3S3RX8HYVD - 


\section{Performance evaluation of classifiers}

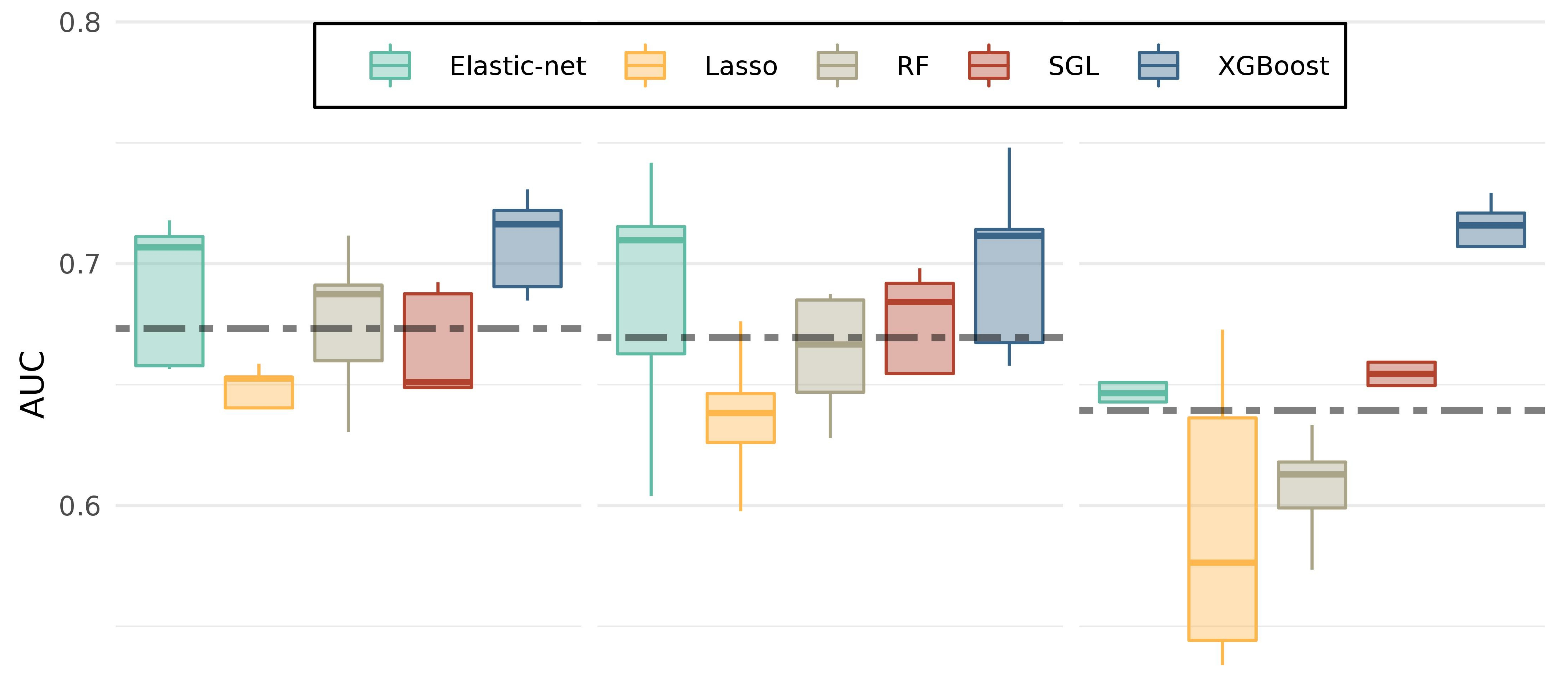

0.5 
A. ROC curves of XGBoost models

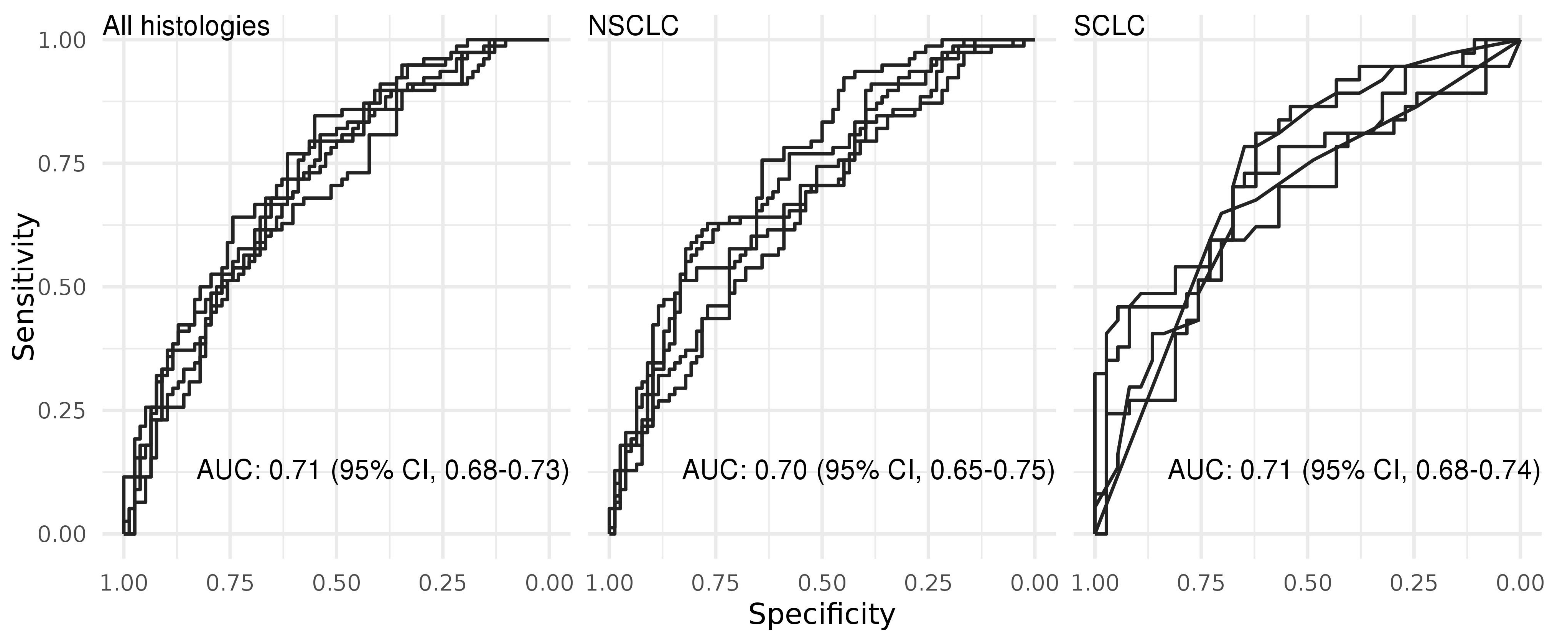

B. ROC curves of XGBoost models (wo serial samples)
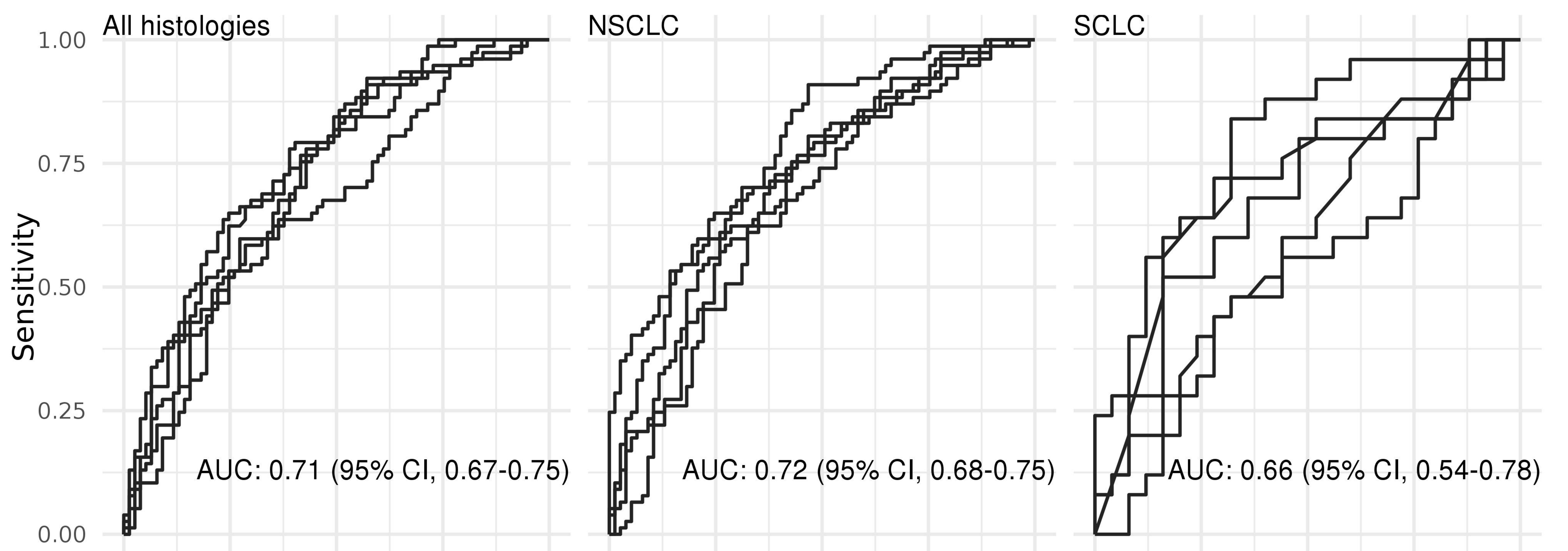
1.00
0.75
0.50
0.25
0.001 .00
0.75 Specificity $^{0.25}$
0.001 .00
0.75
0.50
0.25
0.00 
Prediagnostic models

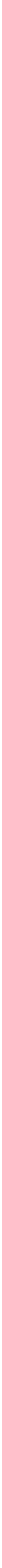


Proposed use of RNA biomarkers

\section{Risk assessment}

Biomarker monitoring of individuals (smokers) with full-time models.

Positive results from any models suggest elevated risk (at least 2 times) while others least concern.

\section{Early detection}

Biomarker monitoring of elevated risk individuals with prediagnostic models. Positive results suggest selection for CT monitoring and a signal between preneoplasia and early stage tumor. Histology specific models can further improve diagnosis accuracy (mean accuracy more than $80 \%$ ). Biyearly monitoring is recommended for others with prediagnostic models. 